\title{
Phyllodes Tumor
}

National Cancer Institute

\section{Source}

National Cancer Institute. Phyllodes Tumor. NCI Thesaurus. Code C2977.

A benign, borderline, or malignant fibroepithelial neoplasm arising from the breast and rarely the prostate gland. It may recur following resection. The recurrence rates are higher for borderline and malignant phyllodes tumors. In borderline and malignant phyllodes tumors metastases to distant anatomic sites can occur. The incidence of metastases is higher in malignant phyllodes tumors. 